\title{
Attention to One or Two Features in Left or Right Visual Field: A Positron Emission Tomography Study
}

\author{
Rik Vandenberghe,, ${ }^{1,5}$ John Duncan, ${ }^{2}$ Patrick Dupont, ${ }^{3}$ Robert Ward, ${ }^{2,6}$ Jean-Baptiste Poline,,$^{5}$ Guy Bormans, ${ }^{3}$ \\ Johan Michiels, ${ }^{4}$ Luc Mortelmans, ${ }^{3}$ and Guy A. Orban ${ }^{1}$ \\ 1 Laboratorium voor Neuro- en Psychofysiologie, Katholieke Universiteit Leuven, Belgium, ${ }^{2}$ Medical Research Council \\ Applied Psychology Unit, Cambridge CB2 2EF, United Kingdom, Departments of ${ }^{3}$ Nuclear Medicine and ${ }^{4}$ Radiology, \\ University Hospital Gasthuisberg, Leuven, Belgium, 5 Wellcome Department of Cognitive Neurology, Institute of Neurology, \\ London WC1N 3BG, United Kingdom, and 'School of Psychology, University of Wales, Bangor, Gwynedd LL57 2DG, \\ United Kingdom
}

\begin{abstract}
In human vision, two features of the same object can be identified concurrently without loss of accuracy. Performance declines, however, when the features belong to different objects in opposite visual fields. We hypothesized that different positron emission tomography activation patterns would reflect these behavioral results. We first delineated an attention network for single discriminations in left or right visual field and then compared this with the activation pattern when subjects divided attention over two features of a single object or over two objects in opposite hemifields. When subjects attended to a single feature, parietal, premotor, and anterior cingulate cortex were activated. These effects were strongest in the right hemisphere and were, remarkably, unaffected by the direction of attention. In contrast, direction of attention affected occipital and frontal activity: right occipital and left lateral frontal activity were higher with attention to the left, whereas right lateral frontal activity was higher with attention to the right. When subjects identified two features of the same object, parietal,
\end{abstract}

premotor, and anterior cingulate activity was enhanced further, predominantly this time in the left hemisphere. Again, there was no direction sensitivity. Direction-sensitive activation of lateral frontal cortex also was increased. Finally, when subjects divided their attention over opposite hemifields, activity in the direction-sensitive occipital and frontal regions fell to a level midway between those seen during exclusively leftward or rightward attention. Thus, the behavioral efficiency with which we attend to multiple features of a single peripheral object is paralleled by enhanced activity in structures generally active during peripheral selective attention as well as in structures that depend on the specific direction of attention, most notably lateral frontal cortex. In addition, in the direction-sensitive regions, dividing attention over hemifields causes a compromise pattern between the extreme levels obtained during unilateral attention.

Key words: human brain mapping; direction of attention; covert attention; superior parietal; pulvinar; prefrontal
Physiologically, visual attention has been conceptualized as a brain state during which activity in widely separated brain areas is organized to enhance processing of the different features of a selected object (Desimone and Duncan, 1995). The behavioral benefit of focused attention on a single object has been demonstrated by comparing dual and single discriminations of features belonging to one or two objects (Duncan, 1993; Egly et al., 1994; Vecera and Farah, 1994). Subjects attend very efficiently to two attributes when they belong to one object (Duncan, 1984). When the attributes belong to two different objects, however, accuracy decreases, especially for the discrimination reported second. This decrease has been demonstrated for many pairs of features, including displacement and orientation (Duncan, 1993) and brightness and orientation (Duncan, 1984), and occurs when the locations of the objects overlap (Duncan, 1984) as well as when the

Received Nov. 19, 1996; revised Feb. 25, 1997; accepted Feb. 28, 1997.

This work was supported by a grant from the Human Frontier Science Program, from the Belgian National Research Council (Grants 9.0007.88 and 3.0043.89), and from the Queen Elizabeth Medical Foundation. R.V. is a research fellow and P.D. a postdoctoral fellow of the Belgian National Research Council. We are grateful to R. S. J. Frackowiak and K. J. Friston for making the SPM software available. We appreciate the help of Chantal Fransen, who trained the subjects, and we thank the technical staff of the Positron Emission Tomography Unit in Leuven for their assistance.

Correspondence should be addressed to Dr. J. Duncan, MRC Applied Psychology Unit, 15 Chaucer Road, Cambridge CB2 2EF, United Kingdom.

Copyright (C) 1997 Society for Neuroscience $0270-6474 / 97 / 173739-12 \$ 05.00 / 0$ objects are in opposite hemifields (Vecera and Farah, 1994). Using positron emission tomography (PET), we investigated the physiological changes underlying these behavioral results for displacement and orientation discrimination concerning left- or right-sided objects.

In an initial step, we determined which areas were involved in discriminations of the feature of a single object in the left or in the right visual field. Event-related potentials (ERP) (Mangun et al., 1993; Heinze et al., 1994) and PET studies (Corbetta et al., 1993; Heinze et al., 1994; Woldorff et al., 1995) have demonstrated higher activity in occipital areas when subjects attend selectively to events occurring in the contralateral hemifield. In addition, right superior parietal activation has been described during shifts of attention in the left and right visual fields, whereas the left intraparietal sulcus has been reported during right field shifts only (Corbetta et al., 1993). Other studies of lateralized attention, however, have not revealed parietal differences with changing direction of attention (Heinze et al., 1994; Woldorff et al., 1995).

In a second step, we related the activation pattern obtained for single discriminations to that obtained when subjects direct their attention to more features of the same object. We considered three nonexclusive hypotheses. First, activations occurring generally during single-feature discriminations could be enhanced during discrimination of two features. This enhancement would be especially compelling when it exceeds the summed responses 


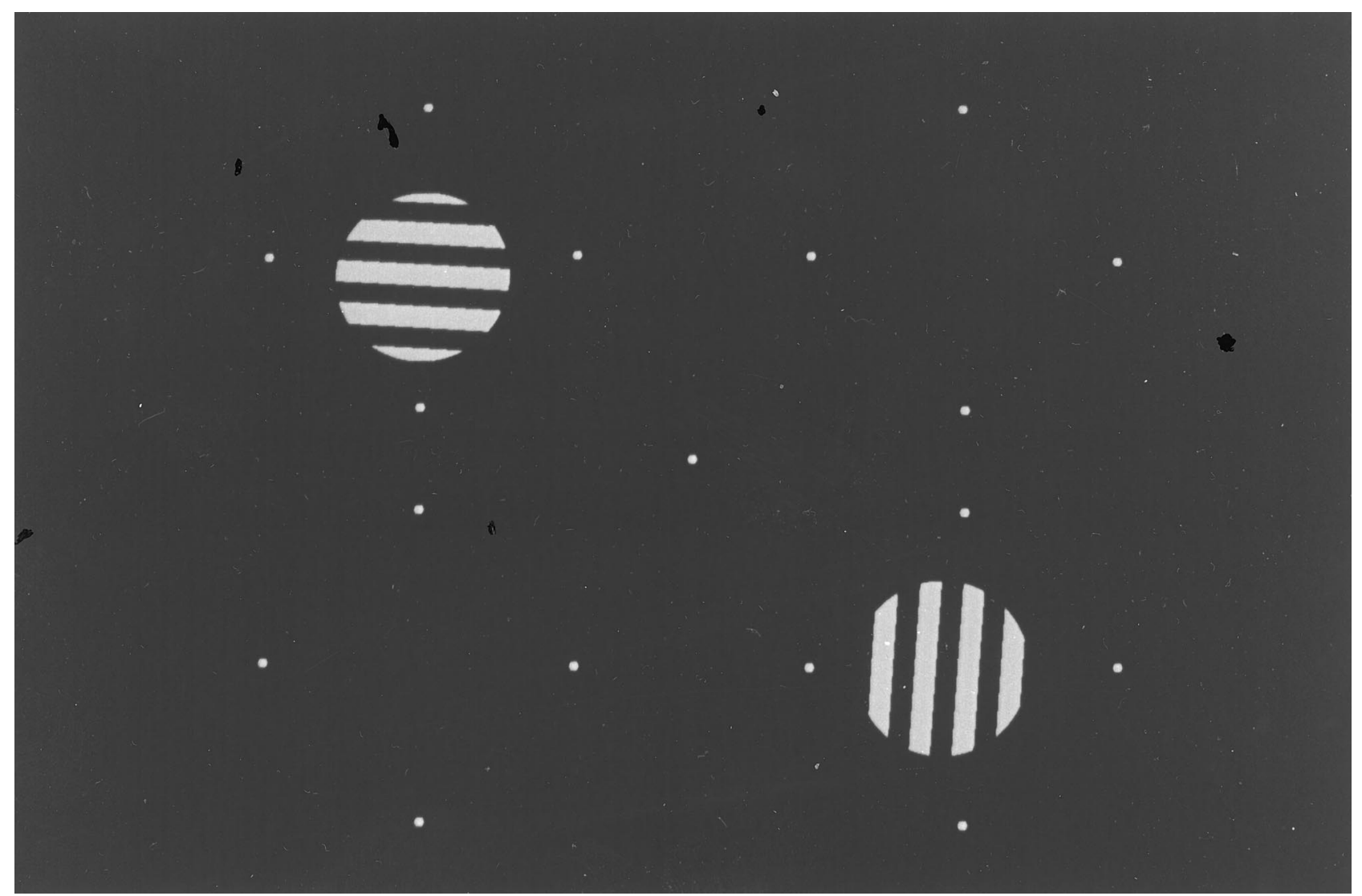

Figure 1. First experiment. Stimulus display, described in detail in the subsection Stimulus Characteristics.

obtained during the component single discriminations. Second, activations that occur during single discriminations specifically when attention is either to the left or to the right could become even more active when subjects attend to two rather than one feature within the favored hemifield. Dual discrimination indeed may require more intense focusing of attention to the contralateral object location than single discrimination does. Third, areas that are specifically more active during single discrimination of either displacement or orientation could be activated in parallel when the two types of attribute are attended concurrently. Attention to displacement might activate components of the occipitoparietal processing pathway and attention to orientation components of the occipitotemporal pathway (Haxby et al., 1994). When one task required attention to the two features, we expected to see integrated activation of these two functionally segregated processing streams.

Finally, we compared conditions in which attention is focused on one object or divided between different objects in opposite hemifields. We hypothesized that the performance decline during divided attention over two objects might be paralleled by weaker patterns of lateralized brain activity (Van Voorhis and Hillyard, 1977) or by recruitment of additional structures because of higher task demands.

\section{MATERIALS AND METHODS}

\section{Subjects}

Fourteen men, aged between 18 and 29 years, participated in the first experiment and 10 men, aged between 20 and 25 years, in the second. All were strictly right-handed, drug-free, had no neurological or psychiatric history, and had a normal brain magnetic resonance image. They gave their written informed consent in accordance with the Declaration of
Helsinki. The experiment was approved by the Ethical Committee of the Medical School, Catholic University of Leuven.

\section{Stimulus characteristics}

Stimuli were displayed using a VISA MC 8521 monitor (width, $14.4^{\circ}$; height $10.7^{\circ}$ ) at a viewing distance of $107 \mathrm{~cm}$ and mounted at an angle of $52^{\circ}$ relative to the horizontal.

First experiment. A central fixation point, accompanied by four-dot "frames" in each visual quadrant, was presented continuously throughout each run, a run being defined as a sequence of consecutive trials (Fig. 1). On each trial two circular grating patches (diameter $2.23^{\circ}$ visual arc, eccentricity $4.45^{\circ}$, spatial frequency 1.79 cycles/degree, phaserandomized) appeared for $495 \mathrm{msec}$, one in the top left and one in the bottom right frame. The left patch was displaced by a specified distance either above or below center within its frame. For any given trial run, the distance of displacement was fixed, while its direction (above or below) varied randomly. This patch also was rotated by a specified angle either clockwise or counterclockwise from horizontal. For any given trial run, the angle of rotation was fixed, while its direction (clockwise or counterclockwise) varied randomly. Similarly, the right patch was displaced by a specified distance either left or right of center and was rotated by a specified angle, either clockwise or counterclockwise from the vertical. An interval of $1656 \mathrm{msec}$ ( \pm random variation of $150 \mathrm{msec}$ ) separated onsets of one display and the next.

Choice of materials was based on a previous behavioral study by Duncan (1993). In that study, as here, patches of lines varied in orientation and displacement with respect to a frame. Results showed a standard contrast between one- and two-object tasks.

Second experiment. Stimuli were similar to those of the first experiment. They were presented for $115 \mathrm{msec}$ with an interval of $2001 \mathrm{msec}( \pm$ random variation of $150 \mathrm{msec}$ ) separating onset of one display and the next. The left grating appeared randomly in the top or bottom quadrant and the right grating in the diagonally opposite quadrant so that left and right visual field stimulation were matched entirely over each run. 
Table 1. First experiment: behavioral parameters

\begin{tabular}{|c|c|c|c|c|c|c|c|c|c|c|c|}
\hline \multicolumn{6}{|c|}{ Stimulus parameters } & \multicolumn{6}{|c|}{$\%$ correct responses } \\
\hline $\begin{array}{l}\text { ldlo } \\
\text { angle }\end{array}$ & disp & $\begin{array}{l}\text { lo } \\
\text { angle }\end{array}$ & $\begin{array}{l}\text { ld } \\
\text { disp }\end{array}$ & $\begin{array}{l}\text { ro } \\
\text { angle }\end{array}$ & $\begin{array}{l}\text { rd } \\
\text { disp }\end{array}$ & $\begin{array}{l}\text { ldlo } \\
\text { lo }\end{array}$ & ld & lo & ld & ro & $\mathrm{rd}$ \\
\hline \pm 1.71 & \pm 0.23 & \pm 1.64 & \pm 0.21 & \pm 1.69 & \pm 0.24 & 85.3 & 89.3 & 89.2 & 88.2 & 85.8 & 84.5 \\
\hline 0.47 & 0.06 & 0.34 & 0.06 & 0.45 & 0.07 & 7.5 & 4.0 & 5.9 & 6.2 & 7.2 & 7.5 \\
\hline
\end{tabular}

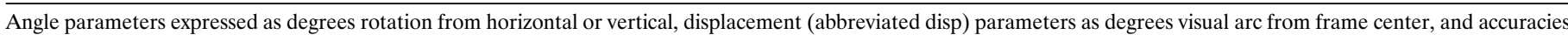
in percentage of correct responses. Top row, group averages; bottom row, SD.

Table 2. Second experiment: behavioral parameters

Stimulus parameters

$\%$ correct responses

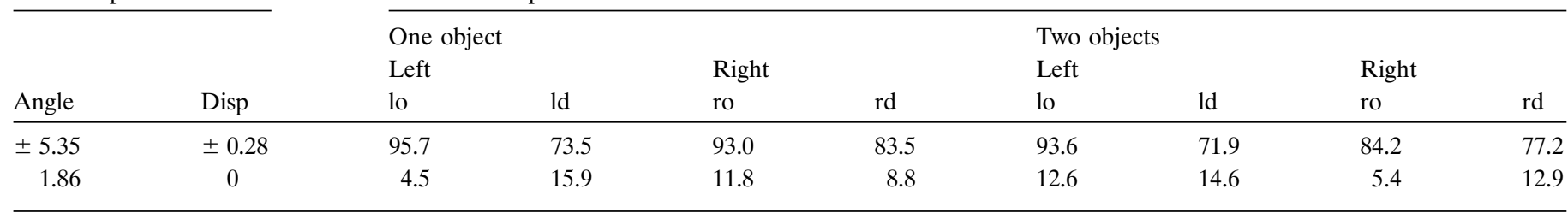

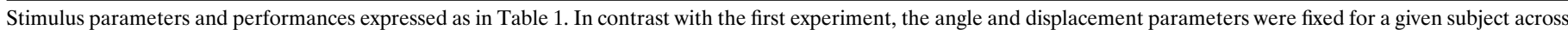

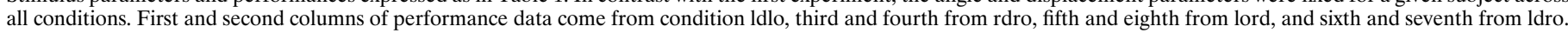
Top row, group averages; bottom row, SD.

\section{Task characteristics}

First experiment. In the first experiment we determined the brain activity pattern during discriminations of single features of left and right objects and related that to the pattern during discrimination of two features of a single object. Because of the limited number of conditions available (6), we could make the latter comparison only for the left visual field.

In the five discrimination conditions, subjects reported, respectively, left displacement and left orientation (ldlo), left orientation (lo), left displacement (ld), right orientation (ro), and right displacement (rd). Each response was a vocal two-alternative forced choice concerning the direction of either displacement or rotation: "boven" (above) versus "onder" (below) for left displacement, "stijgen" (go up) versus "dalen" (go down) for left orientation, "links" (left) versus "rechts" (right) for right displacement, and "stijgen" (go up) versus "dalen" (go down) for right orientation. In ldlo, both displacement and orientation responses were made on each trial, in that order. In single discrimination conditions, a fixed, irrelevant word was spoken after each response, equating total vocal output across conditions. Subjects were trained to respond between the onset of one stimulus and the next. In the control condition (det), the onset of each display was acknowledged simply by a fixed, two-word response. Two days of practice (96 trials/condition per day) were used to train subjects and adapt stimulus parameters (distances of displacement and angles of rotation in each condition) to each individual's performance. PET and behavioral data were gathered on a third day. Mean angle and displacement parameters on this third day are listed in Table 1.

Second experiment. In the second experiment all active tasks required concurrent discrimination of orientation as well as displacement. We compared conditions requiring focused attention to left or right and conditions requiring divided attention between sides.

All four active conditions shared the same type and number of attended attributes. In one experimental condition (ldlo), subjects reported displacement and orientation of the grating on the left, in that order. In a second condition (rdro), subjects reported displacement and orientation of the grating on the right, in that order. In a third condition (ldro), they reported displacement of the left grating and orientation of the right grating, in that order, and in a fourth condition (lord), the orientation of the left grating and the displacement of the right, in that order. Two baseline conditions consisted of passive viewing (pv; no response) and detection (det; fixed two-word response on each trial). Subjects were trained in the same way as during the first experiment. This time, angle and displacement parameters were fixed for a given subject across all conditions; mean values are listed in Table 2.

\section{Recording of eye movements}

Horizontal eye movements were monitored with contact electrodes placed on the outer ocular canthi, with a grounding electrode placed between the eyes. To ensure detection of gaze shifts at the onset or the end of the task, we required subjects to read aloud a digit appearing at the fixation point just before and just after the trial run. Electrooculographical recordings (EOG) were stored on disk. The EOG was calibrated for fixation and for horizontal visually guided saccades of 2 and $4^{\circ}$ amplitude. The EOG was inspected qualitatively for saccades or slow gaze drift occurring during the tasks.

\section{Data acquisition}

The brain was scanned in two-dimensional mode with a PET scanner of the type ECAT931-08-12 (CTI, Knoxville, TN) [voxel size, $x=1.878 \mathrm{~mm}$, $y=1.878 \mathrm{~mm}, z=6.75 \mathrm{~mm}$; transaxial spatial resolution, $8.5 \mathrm{~mm}$ full-width of half-maximum (FWHM); axial spatial resolution, $6.75 \mathrm{~mm}$ FWHM; axial field of view, $10.0 \mathrm{~cm}$ ] (Spinks et al., 1988).

Preparatory phase. Contact electrodes were placed and earphones installed. The subject's head was immobilized with a thermally molded head holder and positioned parallel to the infero-orbitomeatal line by using laser alignment beams. A rectilinear scan was taken for positioning.

Image acquisition. A transmission scan with a germanium-gallium source was taken to correct for attenuation. Each subject was scanned once in each of the six different conditions, each consisting of 64 trials (106 sec). ${ }^{15} \mathrm{O}$-labeled $\mathrm{H}_{2} \mathrm{O}(50 \mathrm{mCi})$ was injected over a period of $12 \mathrm{sec}$ in 20 subjects (first and second experiment) and $40 \mathrm{mCi}$ in the others (first experiment). The task was started at the same instant as the injection. The acquisition began as soon as the intracranial radioactivity count rate rose sharply, i.e., on average 29 (SE 2) sec after the onset of the task. The first $40 \mathrm{sec}$ of image acquisition were used for further analysis. The attenuation-corrected data were reconstructed as 15 planes, using filtered back projection with a Hanning filter of cut-off frequency of 0.5 cycles/ pixel. The brain tissue radiation count rate was used as a measure of regional cerebral blood flow. The task order for the first experiment was counterbalanced with constraints that the detection condition came either third or fourth and that rightward and leftward attention conditions were blocked. The task order for the second experiment was counterbalanced with the constraint that passive viewing and detection came in the middle. An interval of at least 15 min separated two successive injections. In the period between scans, subjects received a 32-trial practice run of the condition to be scanned next.

\section{Individual data}

All subsequent data analysis was performed with Statistical Parametric Mapping, version 1995 (SPM95) (Friston et al., 1995b). Images of all six conditions were available for each subject. The scans from each subject were realigned by using the first image as a reference. They were transformed stereotactically (Friston et al., 1995a) to a standard template in the Talairach space (Talairach and Tournoux, 1988). Images were smoothed with a Gaussian filter of $20 \times 20 \times 12 \mathrm{~mm}^{3}$. 


\section{Group analysis}

First experiment. Data were analyzed using a randomized block design with global brain activity as a covariate of no interest, fixed at $50 \mathrm{ml} / \mathrm{dl} \mathrm{per}$ minute (Friston et al., 1995b).

In the first and the second contrast, we delineated a general selective peripheral attention network involved in covert attention across variations in the type of attribute and in the attended hemifield. We averaged the four single discrimination conditions and compared detection with that average $[\overline{(l o+l d+r o+r d)}-$ det and its inverse] (Table 3 , Figs. $2 A, 3 A$ ).

The subsequent two contrasts allowed us to determine where activity depended on the direction of spatial attention. The mean of the single discriminations of the left grating was compared with the mean of single discriminations of the right grating $[\overline{(l o+l d)}-\overline{(r o+r d)}$ and $\overline{(r o+r d)}$ $-\overline{(l o+l d)}]$ (Table 4, Figs. 2B, 3B,D).

The fifth and sixth contrasts allowed us to identify feature-specific processing areas. We compared the mean of the single orientation discriminations of the left and right grating with the mean of the single displacement discriminations of the left and right grating $[\overline{(l o+r o)}-\overline{(l d}$ $\overline{+r d)}$ and $\overline{(l d+r d)}-\overline{(l o+r o)}]$.

In the seventh contrast, we determined where concurrent discrimination of two features of a left object yielded higher activity than the average of the equivalent single discriminations. We compared concurrent discrimination of orientation and displacement of the left grating with the mean of the single discriminations of orientation and displacement of the left grating [ldlo $-\overline{(l o+l d)}$ (Table 5, Figs. $2 C, 3 C$ ).

In each subtraction we used a significance threshold of $p<0.05$ corrected for multiple comparisons by the standard procedure of SPM95 (Friston et al., 1995b). Activations at a corrected $p<0.2$ also will be mentioned (Poline et al., 1996). Where we wanted to further evaluate negative findings and to determine whether they implied either absent or weak activation, we explored the data at an uncorrected $p<0.01$.

Second experiment. The first experiment provided us with an a priori anatomical hypothesis about the areas specifically involved in concurrent discrimination and about the areas influenced by the direction of attention during single discrimination. In the second experiment we examined how these a priori defined regions responded during concurrent discrimination when subjects focused attention on a single object in the left or in the right hemifield or when they divided their attention over objects appearing on the left and the right.

Brain regions were defined on the basis of the results from the first experiment, using the subtractions ldlo $-\overline{(l d+l o)}, \overline{(l d+l o)}-\overline{(r d+r o)}$ and $\overline{(r d+r o)}-\overline{(l d+l o)}$. Voxels were included when they fulfilled two criteria. The first criterion was identical to that applied in any SPM analysis and excluded all voxels in which, in the second experiment, $\mathrm{rCBF}$ was lower than $80 \%$ of the image maximum rCBF level or the $F$ value did not reach $p<0.05$ in a one-way ANOVA over all conditions. According to our second criterion, we only included voxels for further analysis when they had reached a corrected $p$ value of $p<0.1$ in one of the three above contrasts during the first experiment. These voxels then were grouped in left and in right regions, depending on their coordinates. Then the General Linear Model was applied on the average radioactivity count rates in these regions in exactly the same way as it usually is applied for voxels (Friston et al., 1995b). Data were analyzed using a randomized block design with global brain activity as a covariate of no interest, fixed at $50 \mathrm{ml} / \mathrm{dl}$ per minute (Friston et al., 1995b). $p$ values were corrected by dividing the uncorrected value by the number of regions examined (Bonferroni correction).

\section{RESULTS}

The final image smoothness estimate (FWHM) in the first experiment was $x=21.3, y=24.4, z=18.7 \mathrm{~mm}$ and in the second $x=$ $20.5, y=24.0, z=17.5 \mathrm{~mm}$. The analyzed brain volume in the first experiment extended from $z=-24$ to $+54 \mathrm{~mm}$ anteriorly and -28 to $+48 \mathrm{~mm}$ posteriorly; in the second experiment the volume extended from $z=-10$ to $+54 \mathrm{~mm}$ anteriorly and -32 to +48 $\mathrm{mm}$ posteriorly.

\section{Behavioral parameters}

One subject made several saccades in the scanning session during the orientation discrimination concerning the left grating. All other subjects fixated well in all scanning conditions.
In the first experiment accuracies did not differ significantly between conditions (repeated measures one-way ANOVA $F_{(5,12)}$ $=1.13$ ) (Table 1). A paired $t$ test between the performances on the orientation discriminations during ldlo and during lo, for those subjects who performed both tasks with identical orientation angle parameters, showed no significant difference $(p>0.1)$. The same was true for the displacement discriminations during ldlo and ld $(p>0.4)$. The results of these $t$ tests confirm that subjects perform two-feature and one-feature discriminations with equal accuracy when the two features belong to a single object (Duncan, 1984, 1993).

The accuracies in the second experiment (Table 2) were examined with a three-way ANOVA, with number of objects, attended hemifield, and attended attribute as within-subject factors. There was a main effect of number of objects attended (higher accuracy for one than for two objects, $\left.F_{(1,9)}=69.8, p<0.0000\right)$ and a main effect of attended attribute (higher accuracy for orientation than for displacement, $\left.F_{(1,9)}=17.6, p<0.005\right)$. There was also a significant interaction between number of attended objects and hemifield $\left(F_{(1,9)}=5.76, p<0.05\right)$. In agreement with earlier results (Duncan, 1984, 1993), it was the right-sided object (reported second) that suffered the greatest loss of accuracy in two-object discrimination.

\section{Attention to one feature of a peripheral object (first experiment)}

We determined which areas are involved in discriminations of single features of single objects in the peripheral visual field by subtracting the detection task from the average of the four single discrimination tasks. As shown in Figures $2 A$ and $3 A$, a distributed, mainly right-hemispheric circuit was activated. Activation peaks, together with their rCBF profiles, are listed in Table 3. Note that activations at $p<0.2$ (corrected) are included in the figures, whereas a threshold at $p<0.05$ (corrected) is used for the tables.

This peripheral selective-attention circuit consisted of the right intraparietal sulcus (Figs. $2 A, z=32 \mathrm{~mm} ; 3 A$ ), the right superior parietal lobule (Fig. $2 A, z=44 \mathrm{~mm}$ ), the right upper precentral gyrus (Figs. $2 A, z=44 \mathrm{~mm} ; 3 A$ ), and the right lower precentral sulcus (Fig. $2 A, z=32 \mathrm{~mm}$ ), as well as the left pulvinar (Fig. $2 A, z=8 \mathrm{~mm}$ ).

At $p<0.2$, the right middle frontal gyrus (BA46/10; 34, 38, 20; $Z=4.05$ ) (Figs. $2 A, z=20 \mathrm{~mm} ; 3 A$ ) and the cingulate sulcus (BA32; 8, 8, 44; $Z=4.01$ ) (Fig. $2 A, z=32-44 \mathrm{~mm}$ ) also were activated, together with the right fusiform gyrus (BA19; 50, -70 , $-12 ; Z=4.03$ ) (Fig. $2 A, z=-16 \mathrm{~mm}$ ) and the right middle occipital gyrus (BA19; 34, $-82,16 ; Z=3.60)$, the left intraparietal sulcus (BA40; $-26,-40,32 ; Z=3.76$ ) (Figs. $2 A, z=32 \mathrm{~mm} ; 3 A$ ), as well as the right anterior pallidum $(20,16,4 ; Z=3.68)$ (Fig. $2 A, z=8 \mathrm{~mm})$.

Nearly all of these activations are located in the right hemisphere (Fig. 3A). Does the left hemisphere, apart from the pulvinar and the intraparietal sulcus, contribute anything at all during single discriminations of peripheral objects? To explore this, we lowered the threshold to uncorrected $p<0.01$ for the left hemisphere only. Several activations were found at approximately symmetrical locations to the right-hemispheric activations: in the left middle occipital gyrus (BA18/19; $-34,-88,12 ; Z=2.42$ ), superior parietal cortex $(\mathrm{BA} 7 ;-14,-68,44 ; Z=2.46)$, and precentral sulcus (BA6/44; $-54,6,28 ; Z=3.21)$. This indicates that single discriminations do not rely exclusively on righthemispheric areas despite a clear right-hemispheric dominance. 


\section{A. Single discriminations minus detection}

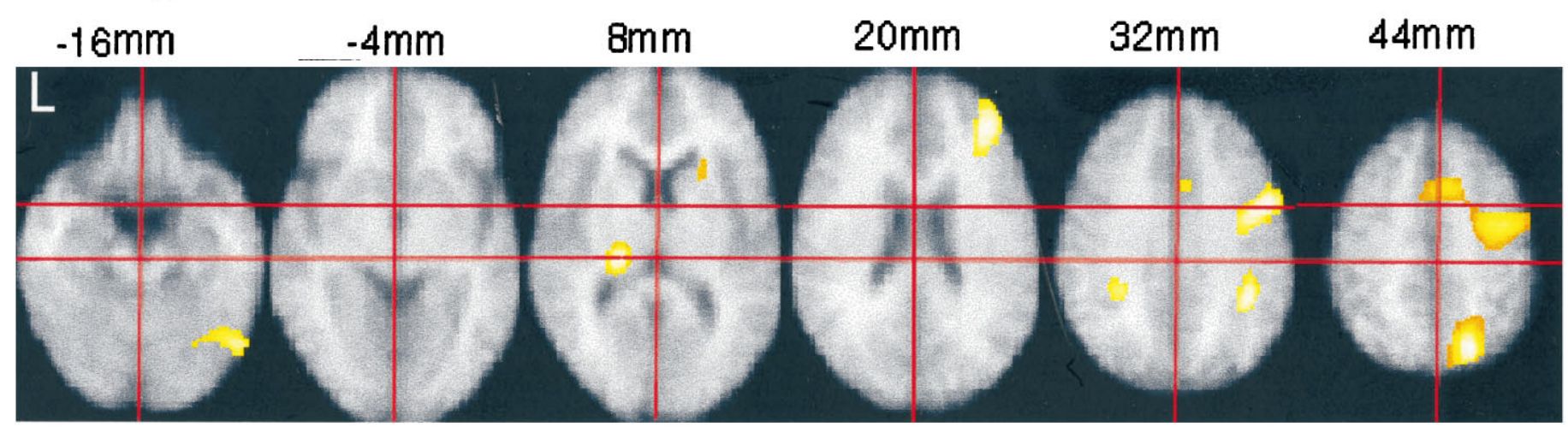

\section{B. Leftward minus rightward attention}

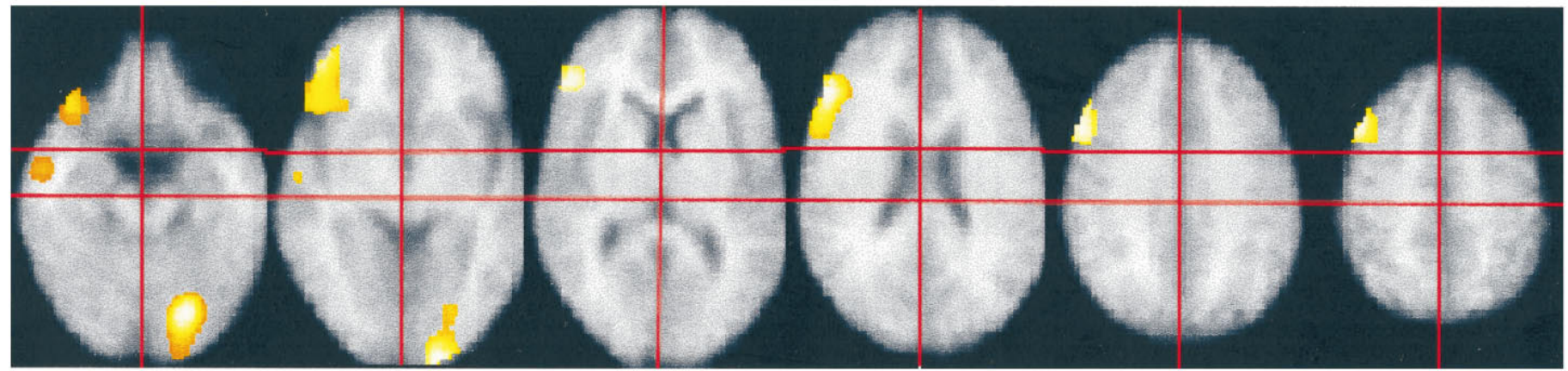

\section{Dual minus single discrimination (leftward attention)}

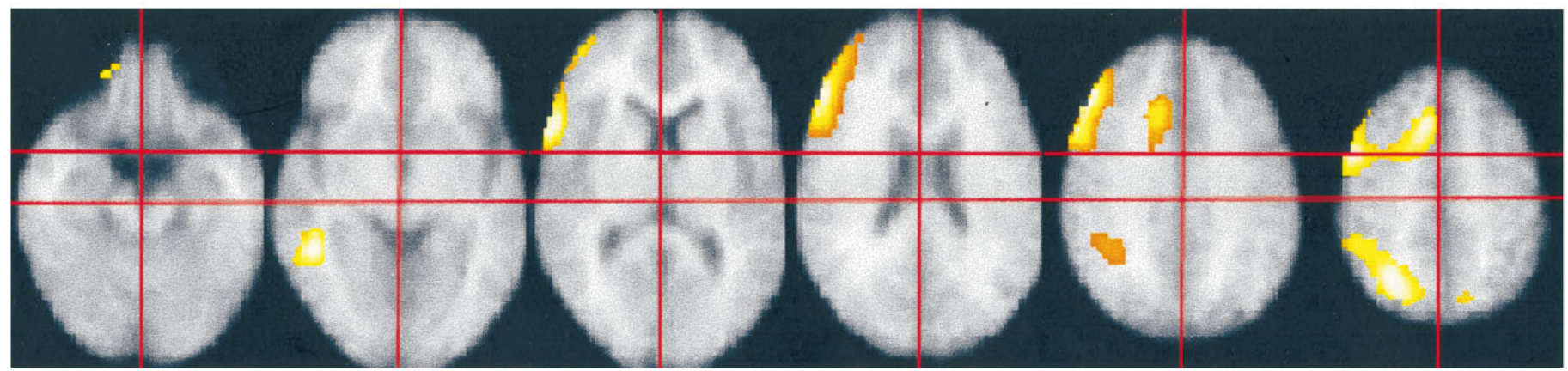

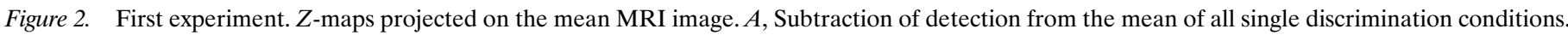

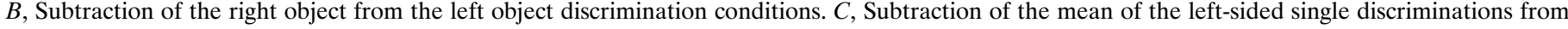

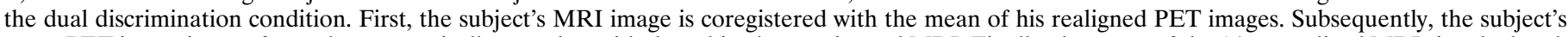

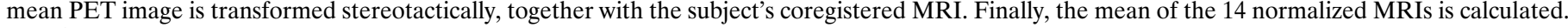
Significance threshold, $p<0.2$.

Several additional low-threshold activations did not correspond to any of the areas that had been activated in the right hemisphere at a corrected $p<0.05$.

Decreases over all active conditions compared with baseline occurred in left anterior fusiform gyrus $(-46,-14,-24 ; Z=$ $4.82)$, left anterior medial frontal gyrus $(-12,58,8 ; Z=4.83)$, left middle temporal gyrus $(-56,-40,-8 ; Z=5.02)$, left superior temporal sulcus $(-52,-60,16 ; Z=5.21)$, and left inferior parietal lobule $(-42,-70,32 ; Z=4.95)$. This pattern of decreases in active, as compared with more passive, conditions is consistent with what has been described previously over a wide variety of tasks (Shulman et al., 1996).

\section{Single discriminations: effect of direction of attention (first experiment)}

Occipital cortex

When subjects attended to the left instead of the right, the right lingual gyrus (Table 4, Fig. $2 B, z=-16$ to $-4 \mathrm{~mm}$ ) was significantly more active, together with the right calcarine (Table 4). This effect extended into the right posterior fusiform site described under the general network $(50,-70,-12 ; Z=2.62)$.

No equivalent effect was observed on the left side during rightward attention, even when the threshold was lowered to an uncorrected $p<0.01$. 


\section{A. Single discriminations minus detection}
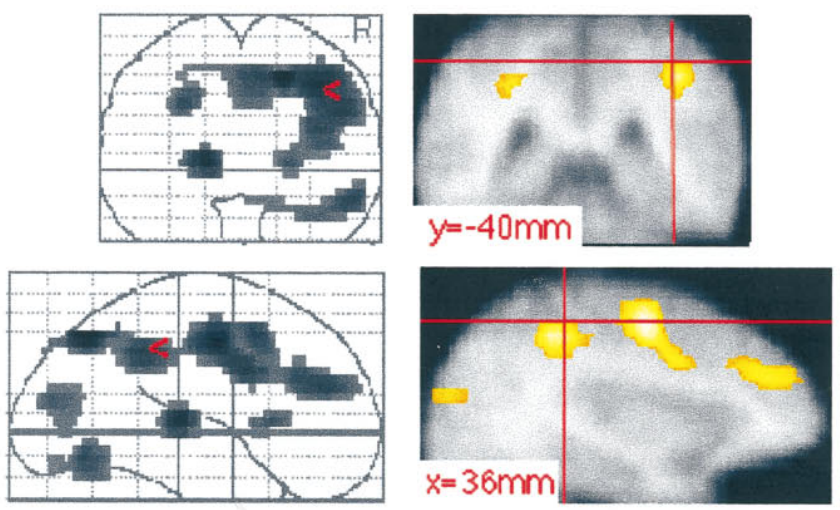

\section{B. Leftward minus rightward attention}
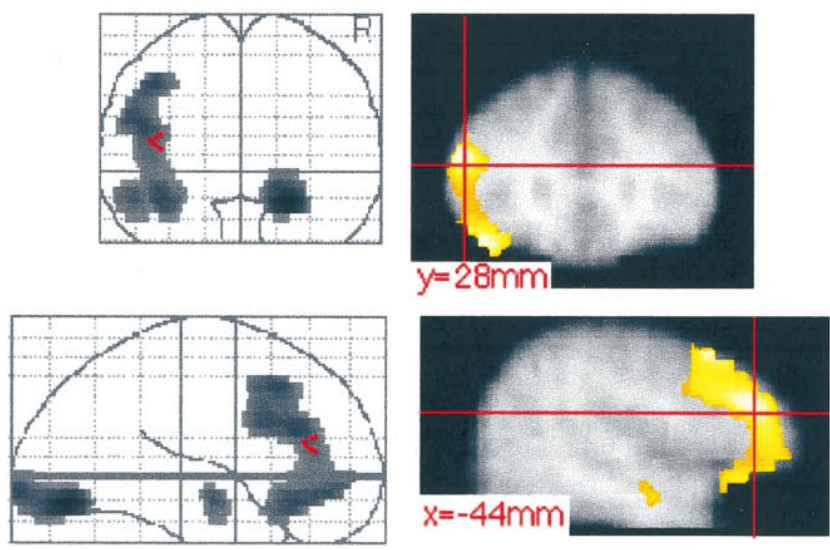

\section{Dual minus single discrimination (leftward attention)}
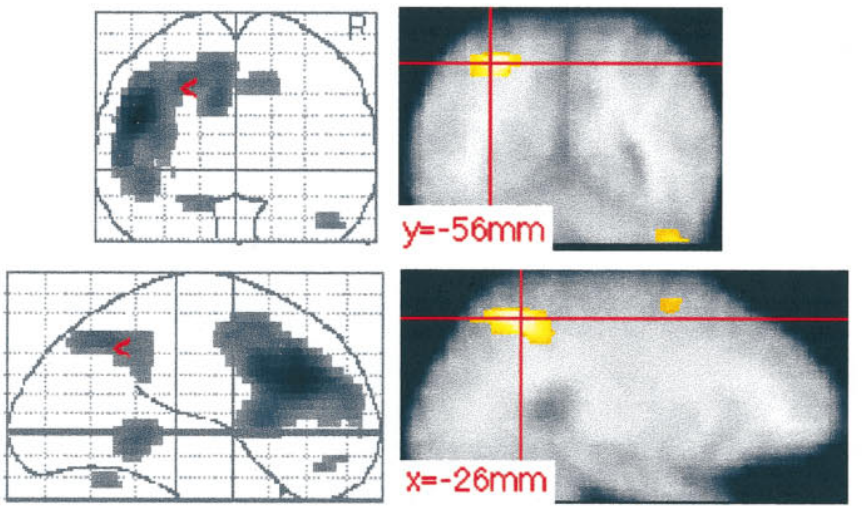

\section{Rightward minus leftward attention}
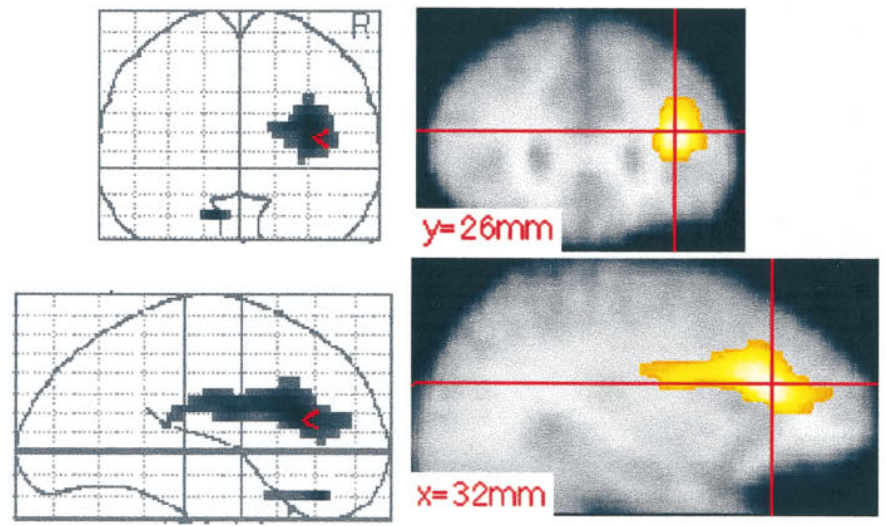

Figure 3. First experiment. All $Z$-maps are thresholded at $p<0.2$. The right hemisphere is on the reader's right hand in the coronal views. $A$, Subtraction of detection from the mean of all single discrimination conditions. Left top and bottom subquadrants, Coronal and sagittal see-through projections; right top subquadrant, coronal slice through $y=-40 \mathrm{~mm}$; left bottom subquadrant, sagittal slice through $x=36 \mathrm{~mm}$. The crosshair marks the right intraparietal sulcus. $B$, Subtraction of the right object from the left object single-feature discrimination conditions. Left top and bottom subquadrants, Coronal and sagittal see-through projections; right top subquadrant, coronal slice through $y=28 \mathrm{~mm}$; left bottom subquadrant, sagittal slice through $x=-44 \mathrm{~mm}$. The crosshair marks the left lateral frontal activation. $C$, Subtraction of the mean of the single discriminations from the dual discrimination condition. Left top and bottom subquadrants, Coronal and sagittal see-through projections; right top subquadrant, coronal slice through $y=-56 \mathrm{~mm}$; right bottom subquadrant, sagittal slice through $x=-26 \mathrm{~mm}$. The crosshair marks the left superior parietal lobule. $D$, Subtraction of the left object from the right object single-feature discriminations. Left top and bottom subquadrants, Coronal and sagittal see-through projections; right top subquadrant, coronal slice through $y=26 \mathrm{~mm}$; right bottom subquadrant, sagittal slice through $x=32 \mathrm{~mm}$. The crosshair marks the right lateral frontal activation.

\section{Parietal cortex}

Remarkably, the direction of attention had no significant influence on parietal activity. To explore this negative finding further, we used two strategies.

First, we probed the parietal voxels obtained in the subtraction of detection from the averaged single discriminations. Activity in the right superior parietal voxel at $18,-68,44$ (Table 3 ) or at the peak in the right intraparietal sulcus at $36,-42,36$ (Table 3 ) did not differ between leftward and rightward attention even at an uncorrected $p<0.01$. Certainly, Table 3 suggests no trend toward higher activity during contralateral attention.

Second, we explored the brain activations in the parietal cortex at a threshold $p<0.01$ (uncorrected). Attention to the left instead of the right produced higher activity at the junction between occipital and parietal cortex (BA19/39), both in the left hemisphere $(-34,-66,20 ; Z=3.33$; percentage $\mathrm{rCBF}$ difference with respect to baseline: ldlo $-0.03 \%$, lo $0.13 \%$, ld $-0.60 \%$, ro $-1.98 \%$, rd $-1.28 \%)$ and in the right $(44,-66,36$; $Z=2.59$; ldlo $-3.13 \%$, lo $-1.12 \%$, ld $-0.51 \%$, ro $-2.66 \%$, rd $-1.65 \%)$. When attention was to the right instead of the left, activity increased in the right inferior parietal lobule (BA39/40) $(40,-46,28 ; Z=2.53$; ldlo $1.45 \%$, lo $0.09 \%$, ld $1.60 \%$, ro $2.22 \%$, rd $1.87 \%$ ). No strong conclusions can be drawn from these nonsignificant activations.

\section{Frontal cortex}

Substantial areas of left lateral frontal cortex were more active during attention to the left than during attention to the right (Figs. $2 B, z=-16$ to $44 \mathrm{~mm} ; 3 B$, Table 4$)$. Conversely, right inferior frontal sulcus (BA44/45) was more active when attention was directed to the right instead of the left (Fig. 3D, Table 4). At $p<$ 0.2 , subtracting leftward from rightward attention revealed a further activation in the left orbital gyri $(-12,36,-20 ; Z=3.94)$.

\section{Temporal cortex}

As shown in Table 4, a region in the left middle temporal gyrus (Figs. $2 B, 3 B$ ) was more active during leftward than during rightward attention. This area fell within an area of net deactivation in the general circuit. 
Table 3. General circuit for peripheral single discrimination: \% rCBF increases

\begin{tabular}{|c|c|c|c|c|c|c|c|c|}
\hline & & $\begin{array}{l}\text { ldlo- } \\
\text { det }\end{array}$ & $\begin{array}{l}\text { lo- } \\
\text { det }\end{array}$ & $\begin{array}{l}\text { ld- } \\
\text { det }\end{array}$ & $\begin{array}{l}\text { ro- } \\
\text { det }\end{array}$ & $\begin{array}{l}\text { rd- } \\
\text { det }\end{array}$ & $\begin{array}{l}\overline{\text { single }}- \\
\text { det }\end{array}$ & $Z$ \\
\hline $\begin{array}{l}\text { R LPs } \\
\text { BA7 }\end{array}$ & $18,-68,44$ & 5.49 & 3.10 & 4.05 & 2.83 & 4.09 & 3.91 & 5.08 \\
\hline $\begin{array}{l}\text { R IPS } \\
\text { BA40 }\end{array}$ & $36,-42,36$ & 4.39 & 1.43 & 3.88 & 3.21 & 3.55 & 3.01 & 4.39 \\
\hline $\begin{array}{l}\mathrm{R} \text { upper GPrC } \\
\text { BA6 }\end{array}$ & $34,-8,40$ & 3.84 & 1.94 & 3.30 & 2.44 & 3.58 & 2.81 & 4.70 \\
\hline $\begin{array}{l}\text { R lower PrCS } \\
\text { BA6/44 }\end{array}$ & $44,4,28$ & 2.34 & 1.91 & 2.68 & 2.65 & 3.00 & 2.52 & 4.31 \\
\hline $\mathrm{L}$ pulvinar & $-18,-26,4$ & 3.78 & 3.66 & 3.11 & 3.74 & 3.50 & 3.56 & 4.66 \\
\hline
\end{tabular}

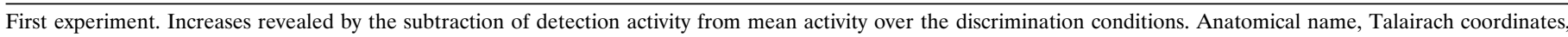

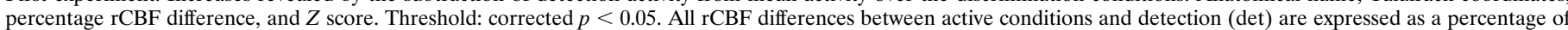

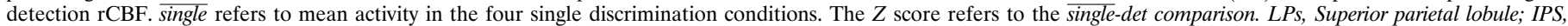
intraparietal sulcus; GPrC, precentral gyrus; PrCS, precentral sulcus.

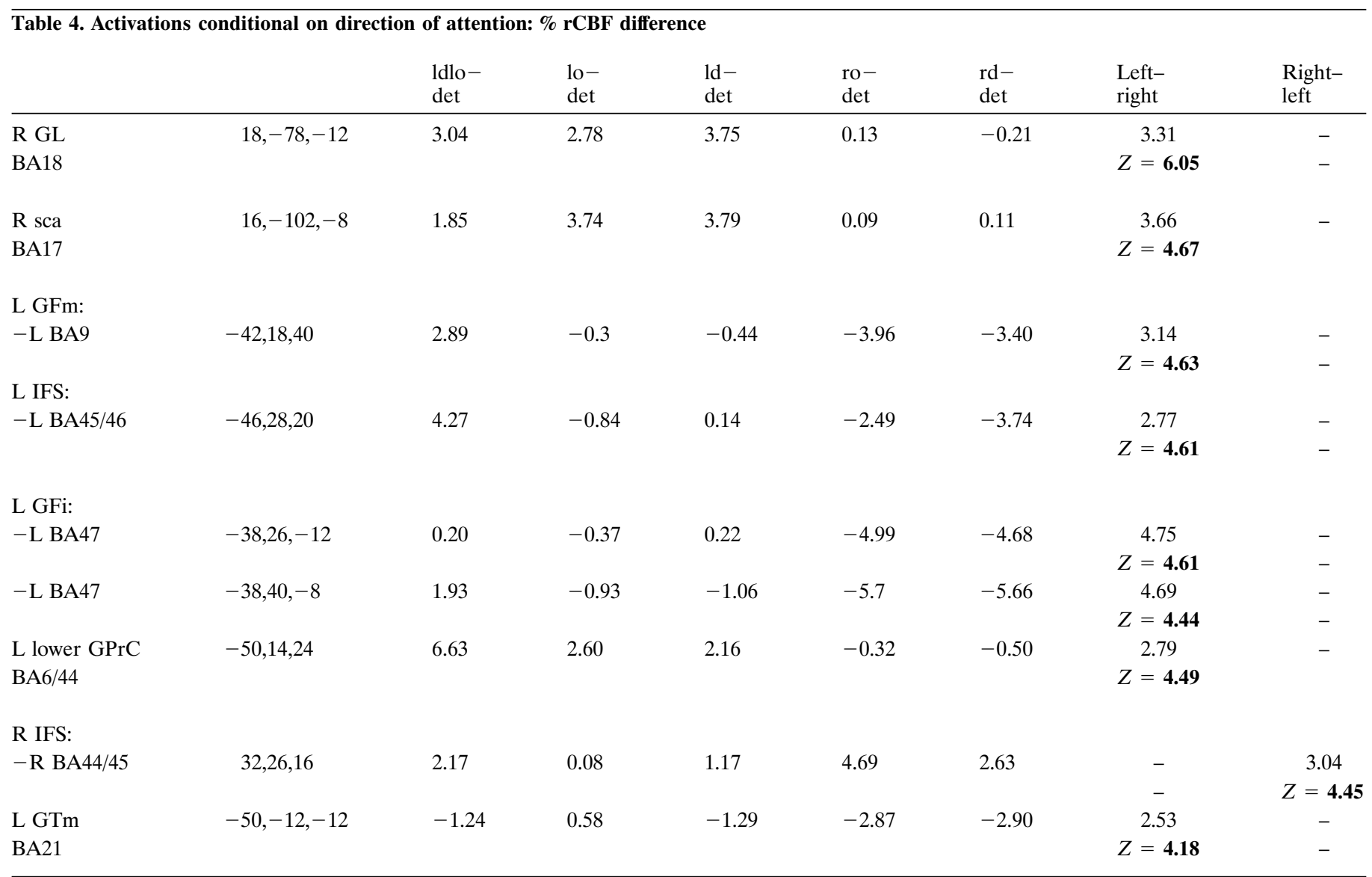

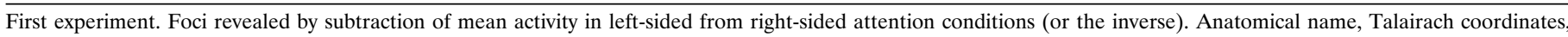

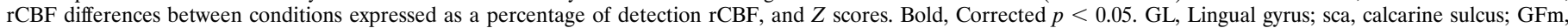
middle frontal gyrus; GFi, inferior frontal gyrus; IFS, inferior frontal sulcus; GTm, middle temporal gyrus. See previous table for other abbreviations.

\section{Single discriminations: effect of type of attended attribute (first experiment)}

We determined the areas specifically involved in selective attention to orientation or to displacement by contrasting the average of the orientation discriminations with the average of the displacement discriminations.

\section{Occipital cortex}

The left anterior ventral occipital cortex $(-42,-44,-20$, BA36/ $37 ; Z=4.35$ ), at the border between fusiform and parahippocampal gyrus, was more active when subjects attended to the orientation of the grating than when they attended to its displacement. This difference resulted from both an increase during orientation 


\begin{tabular}{|c|c|c|c|c|c|c|c|}
\hline & & $\begin{array}{l}\text { ldlo- } \\
\text { det }\end{array}$ & $\begin{array}{l}\text { lo- } \\
\text { det }\end{array}$ & $\begin{array}{l}\text { ld- } \\
\text { det }\end{array}$ & $\begin{array}{l}\text { ro- } \\
\text { det }\end{array}$ & $\begin{array}{l}\text { rd- } \\
\text { det }\end{array}$ & $\frac{\text { ldlo- }}{(l d+l o)}$ \\
\hline L LPs & \multirow[t]{2}{*}{$-24,-56,40$} & \multirow[t]{2}{*}{4.06} & \multirow[t]{2}{*}{1.37} & \multirow[t]{2}{*}{1.05} & \multirow[t]{2}{*}{1.28} & \multirow[t]{2}{*}{1.64} & 2.85 \\
\hline BA7 & & & & & & & $Z=4.62$ \\
\hline L IFS & \multirow[t]{2}{*}{$-46,30,24$} & \multirow[t]{2}{*}{5.72} & \multirow[t]{2}{*}{-0.27} & \multirow[t]{2}{*}{0.74} & \multirow[t]{2}{*}{-1.72} & \multirow[t]{2}{*}{-3.19} & 5.49 \\
\hline BA46 & & & & & & & $Z=\mathbf{6 . 5 3}$ \\
\hline L lower GPrC & \multirow{2}{*}{$-52,14,8$} & \multirow[t]{2}{*}{3.99} & \multirow[t]{2}{*}{-0.63} & \multirow[t]{2}{*}{0.13} & \multirow[t]{2}{*}{-1.14} & \multirow[t]{2}{*}{-1.23} & 4.25 \\
\hline BA6/44 & & & & & & & $Z=\mathbf{4 . 7 0}$ \\
\hline L upper GPrC & \multirow[t]{2}{*}{$-44,0,44$} & \multirow[t]{2}{*}{4.66} & \multirow[t]{2}{*}{0.99} & \multirow[t]{2}{*}{1.85} & \multirow[t]{2}{*}{-0.37} & \multirow[t]{2}{*}{0.75} & 3.24 \\
\hline BA6 & & & & & & & $Z=4.27$ \\
\hline GC & \multirow[t]{2}{*}{$-10,20,32$} & \multirow[t]{2}{*}{4.80} & \multirow[t]{2}{*}{0.79} & \multirow[t]{2}{*}{1.83} & \multirow[t]{2}{*}{1.22} & \multirow[t]{2}{*}{1.14} & 3.49 \\
\hline BA32 & & & & & & & $Z=\mathbf{4 . 6 5}$ \\
\hline L GTm & \multirow[t]{2}{*}{$-42,-46,-4$} & \multirow[t]{2}{*}{1.34} & \multirow[t]{2}{*}{-1.44} & \multirow[t]{2}{*}{-2.75} & \multirow[t]{2}{*}{-1.18} & \multirow[t]{2}{*}{-1.09} & 3.44 \\
\hline BA21/37 & & & & & & & $Z=\mathbf{4 . 4 2}$ \\
\hline
\end{tabular}

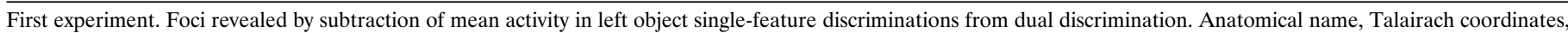

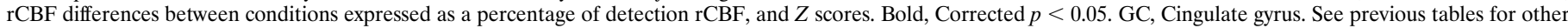
abbreviations.

discrimination, as compared with baseline, and a decrease during displacement discrimination, as compared with baseline. A corresponding contralateral activation $(36,-34,-12)$ fell just below the significance threshold ( $p=0.06, Z=4.02$ ). Another result bordering on significance also is worth noting: the right middle occipital gyrus (44, -76, 4; BA19/37) was activated more strongly during displacement than during orientation discrimination $(p=$ 0.05, $Z=4.06$ ).

\section{Dividing attention over two features of a single object (first experiment)}

We determined which areas were more active during discrimination of two features, as compared with single discriminations, by contrasting the condition in which subjects attended concurrently to orientation and displacement with the averaged conditions in which subjects attended to either orientation or displacement. This comparison could be made for the left visual field only.

As mentioned above, the brain activation pattern during single discriminations (Fig. 2A), as compared with detection, was lateralized mainly to the right. Subtracting this pattern from the activation pattern during dual discrimination left us with a mainly left-hemispheric activation pattern (Figs. 2C, 3C).

The activation pattern obtained from the subtraction of single from dual discriminations (Fig. $2 C$ ) partly consisted of enhanced activity of areas active $(p<0.2)$ during single discriminations (Fig. $2 A$ ): the anterior cingulate (Fig. $2 A, z=32-44 \mathrm{~mm}$ ), the right superior parietal cortex (Fig. $2 A, z=44 \mathrm{~mm}$ ), and the left intraparietal sulcus (Fig. $2 A, z=32 \mathrm{~mm}$ ). This was combined with enhanced activation of specific direction-sensitive areas: left lateral frontal cortex (Fig. 2B, $z=8-44 \mathrm{~mm}$ ). Finally, activity in the left superior parietal lobule, which was weak during single discrimination $(Z=2.46$, see above), was much enhanced during dual discrimination (Fig. $2 C, z=44 \mathrm{~mm}$ ).

\section{Parietal cortex}

The left superior parietal activation during concurrent discrimination (Figs. $2 C, z=44 \mathrm{~mm} ; 3 C$ ) substantially exceeded the sum of the weak rCBF increases observed during the component single discriminations (Table 5). This activation extended into the left intraparietal sulcus (Fig. $2 C, z=32 \mathrm{~mm}$ ).

At $p<0.2$, we observed an additional activation peak in the right medial superior parietal lobule (12, $-70,40 ; Z=3.84$ ) (Fig. $2 C$ ).

\section{Frontal cortex}

As detailed above, a substantial area of left lateral frontal cortex was more active during leftward than during rightward attention (Figs. $2 B, 3 B$ ). Activity in this area increased even further with two concurrent left-field discriminations (Figs. 2C, 3C, Table 5).

\section{Temporal cortex}

An area in the left middle temporal gyrus was activated during concurrent discrimination with respect to single discrimination (Fig. $2 C, z=-4 \mathrm{~mm}$; Table 5). As the comparison between Figure $2 B$ $(z=-16 \mathrm{~mm})$ and Figure $2 C(z=-4 \mathrm{~mm})$ shows, this temporal area lay posterior to the temporal activation obtained in the subtraction of rightward attention from leftward attention. Again, this region fell within an area of net deactivation in the general circuit.

\section{Second experiment: a priori definition of the regions}

For analysis of the second experiment, we included only voxels that, in the first experiment, had revealed a significant (corrected $p<0.1$ ) difference between dual and the averaged single discriminations or between right and left object single discrimination. They were grouped as six regions, using Talairach coordinates of the voxels: right ventral occipital $(x>0, y<-55, z<0)$, left parietal $(x<0, y<-20, z>18)$, left anterior cingulate $(x<0, x>$ $-15, y>-5)$, left lateral frontal $(x<0, x<-20, y>-5)$, right lateral frontal $(x>0, x<-20, y>-5)$, and left posterior temporal $(x<0, y<-20, y>-55, z<0)$. The latter temporal region corresponds to the temporal activation revealed by the subtraction of single from dual discrimination (Fig. 2C). The anterior temporal direction-sensitive voxels shown in Figure $2 B$ did not reach a significant $F$ value in the second experiment, and there was, therefore, no corresponding region in the second study. 
This could be attributable to the restricted volume scanned at lower levels.

We also wanted to confirm the absence of any rCBF differences in the left ventral occipital and right parietal cortex when conditions with rightward and leftward attention were compared. We obtained a left ventral occipital region by mirroring the right ventral occipital area to the other hemisphere and a parietal region by mirroring the left parietal region. The eight resulting regions are displayed in Figure 4.

\section{Dual discrimination: effect of direction of attention (second experiment) \\ Occipital cortex}

As in the first experiment, right ventral occipital $\mathrm{rCBF}$ was highest when attention was toward the object on the left and at its lowest level when attention was toward the object on the right (Table 6). The difference between exclusively rightward and exclusively leftward attention was highly significant (corrected $p<0.005$ ).

The mirrored left ventral occipital region again demonstrated no activation during rightward, as compared with leftward, attention (Table 6), and regional blood flow was even slightly higher during leftward attention. In the first experiment the absence of left ventral occipital modulation could have been explained by the position of the right stimulus in the bottom quadrant, as opposed to the position of the left stimulus in the top quadrant (Woldorff et al., 1996). For the second experiment this explanation is unsatisfactory, because stimuli on either side appeared with equal frequency in the top or bottom quadrant.

\section{Parietal cortex}

Neither left nor right superior parietal blood flow changed with varying direction of attention (Table 6).

\section{Frontal cortex}

In left lateral frontal cortex, as compared with baseline conditions ( $p v$ and det), blood flow was increased during leftward attention and decreased during rightward attention. Similar to the findings in the first experiment, the difference between left object and right object discrimination was highly significant (corrected $p<0.005$ ) (Table 6).

The a priori defined right inferior frontal region was not influenced significantly by the direction of attention (Table 6). When we examined the data from the second experiment without a priori selection criteria, however, a weak right inferior frontal activation $(42,32,-8 ; Z=3.13)$, lying below the predefined region, was observed during attention to the right $(\% \mathrm{rCBF}$ difference: ldlo - det, $-4.12 \%$; rdro - det, $0.35 \%$; lord - det, $-1.91 \%$; ldro - det, $-1.84 \%$ ). Because the lowest anterior brain level scanned was at $z=-10 \mathrm{~mm}$, we cannot exclude any direction effect occurring below that level.

As in the first experiment, anterior cingulate $\mathrm{rCBF}$ was higher during concurrent discrimination than during detection. It was not influenced by the direction of attention (Table 6).

\section{Dividing attention over two hemifields (second experiment)}

When we examined the data from the second experiment without any a priori selection and contrasted the average of single- with the average of dual-object conditions [subtraction (ldlo + rdro) - (ldro + lord)], we found no significant (corrected $p<0.2$ ) differences.

\section{Occipital and frontal cortex}

When subjects divided their attention between objects in the two visual hemifields, blood flow in the a priori defined right occipital

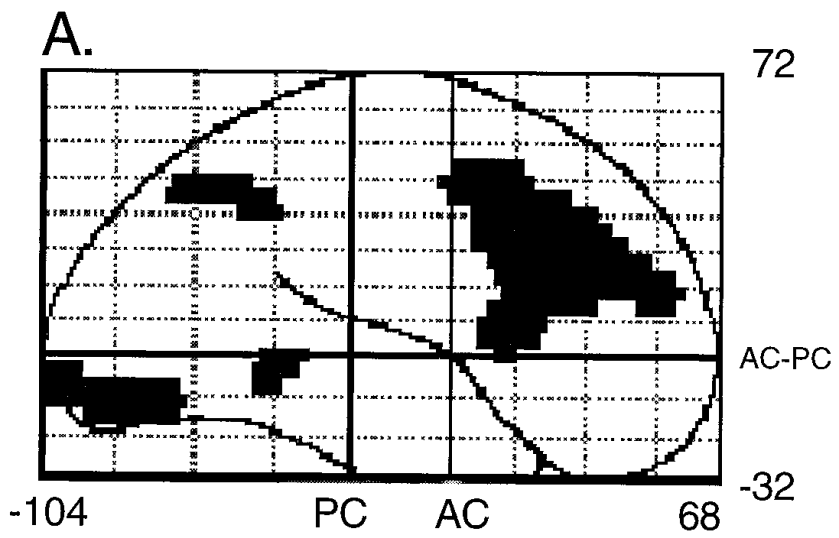

B.
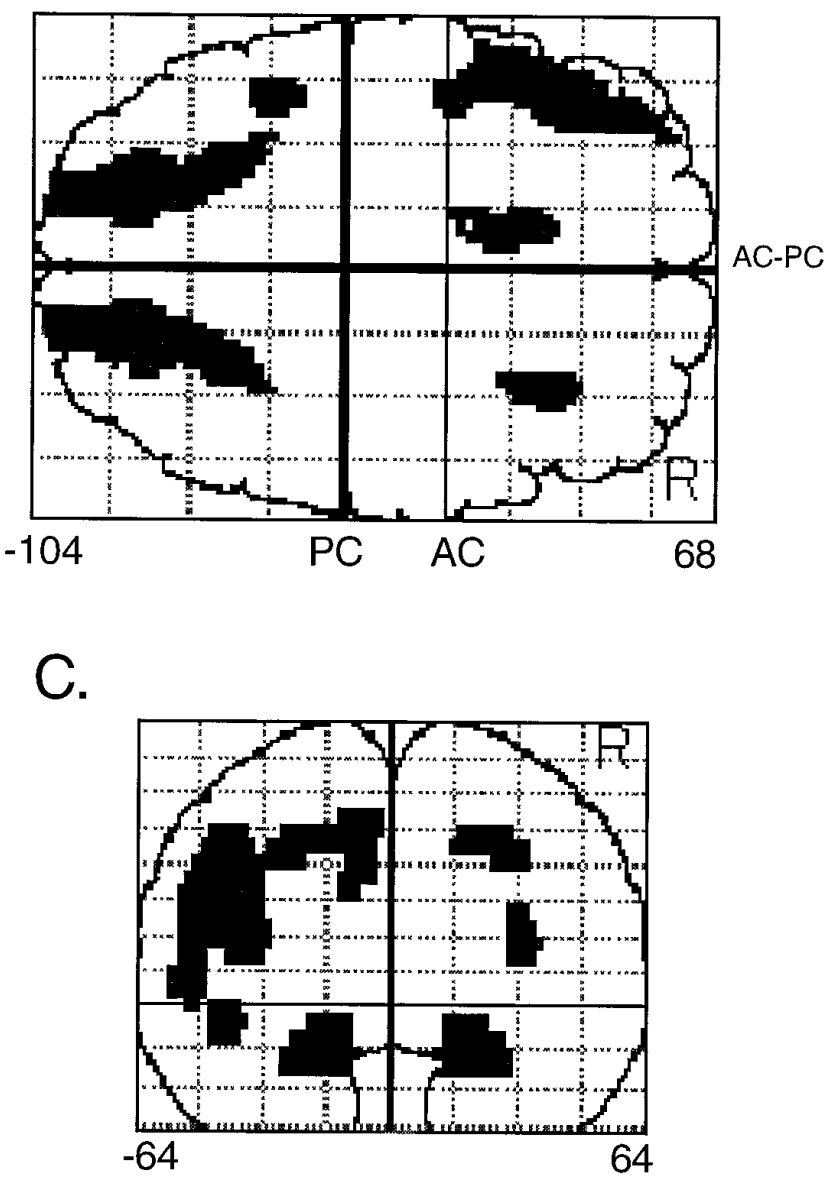

Figure 4. Second experiment. Localization and extent of the a priori defined regions. $A$, Sagittal see-through projection. $B$, Transverse projection. $C$, Coronal projection. The averaged coordinates of the voxels included in these regions follow. Left and right occipital: $x=|19|, y=-84$, $z=-11$; left and right parietal: $x=|24|, y=-58, z=41$; anterior cingulate: $x=-9, y=15, z=39$; left lateral frontal: $x=-44, y=22, z=$ 24; right lateral frontal: $x=31, y=23, z=18$; left temporal: $x=-42, y=$ $-45, z=-3$.

and left lateral frontal regions lay in between the more extreme levels obtained during exclusively leftward or rightward attention (Table 6). The classical subtraction (ldlo + rdro) - (ldro + lord) had overlooked this effect as it failed to show that the component conditions during focused attention (ldlo and rdro) yielded blood 


\begin{tabular}{|c|c|c|c|c|c|c|c|}
\hline & $\begin{array}{l}\text { ldlo- } \\
\text { det }\end{array}$ & $\begin{array}{l}\text { rdro- } \\
\text { det }\end{array}$ & $\begin{array}{l}\text { ldro- } \\
\text { det }\end{array}$ & $\begin{array}{l}\text { lord- } \\
\text { det }\end{array}$ & $\begin{array}{l}\text { pv- } \\
\text { det }\end{array}$ & $\frac{\overline{(l d l o+r d r o)}}{(\text { ldro }+r d l o)}-$ & $\begin{array}{l}\text { ldlo- } \\
\text { rdro }\end{array}$ \\
\hline L occipital & $\begin{array}{l}0.90 \\
Z=1.81\end{array}$ & 0.77 & 0.55 & 0.27 & -0.92 & 0.43 & 0.13 \\
\hline R occipital & $\begin{array}{l}1.96 \\
Z=\mathbf{3 . 6 5}\end{array}$ & 0.08 & $\begin{array}{l}1.04 \\
Z=2.05\end{array}$ & 0.29 & -0.53 & 0.27 & $\begin{array}{l}2.05 \\
Z=\mathbf{3 . 7 3}\end{array}$ \\
\hline $\mathrm{L}$ parietal & $\begin{array}{l}1.54 \\
Z=\mathbf{2 . 8 5}\end{array}$ & $\begin{array}{l}1.46 \\
Z=\mathbf{2 . 6 1}\end{array}$ & $\begin{array}{l}1.69 \\
Z=\mathbf{3 . 1 0}\end{array}$ & $\begin{array}{l}1.73 \\
Z=\mathbf{2 . 9 7}\end{array}$ & -0.68 & -0.21 & 0.08 \\
\hline $\mathrm{R}$ parietal & $\begin{array}{l}1.67 \\
Z=\mathbf{3 . 4 3}\end{array}$ & $\begin{array}{l}1.11 \\
Z=2.19\end{array}$ & $\begin{array}{l}0.81 \\
Z=1.67\end{array}$ & $\begin{array}{l}1.11 \\
Z=\mathbf{3 . 5 1}\end{array}$ & -0.52 & 0.07 & 0.56 \\
\hline $\mathrm{L}$ ant. cingulate & $\begin{array}{l}1.70 \\
Z=\mathbf{2 . 9 9}\end{array}$ & $\begin{array}{l}1.34 \\
Z=2.30\end{array}$ & 0.52 & $\begin{array}{l}1.41 \\
Z=2.36\end{array}$ & -0.50 & 0.55 & 0.36 \\
\hline L lat. frontal & $\begin{array}{l}0.65 \\
Z=2.01\end{array}$ & -0.64 & -0.02 & -0.17 & -0.16 & 0.10 & $\begin{array}{l}1.29 \\
Z=\mathbf{3 . 6 9}\end{array}$ \\
\hline $\mathrm{R}$ lat. frontal & $\begin{array}{l}1.75 \\
Z=\mathbf{3 . 7 7}\end{array}$ & $\begin{array}{l}0.96 \\
Z=2.12\end{array}$ & $\begin{array}{l}1.00 \\
Z=\mathbf{3 . 1 9}\end{array}$ & $\begin{array}{l}1.54 \\
Z=2.28\end{array}$ & $\begin{array}{l}0.84 \\
Z=1.79\end{array}$ & 0.09 & $\begin{array}{l}0.78 \\
Z=1.77\end{array}$ \\
\hline $\mathrm{L}$ temporal & -1.35 & -0.98 & -0.84 & -0.53 & -1.39 & -0.48 & -0.37 \\
\hline
\end{tabular}

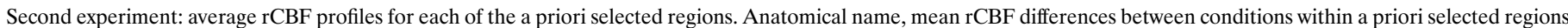

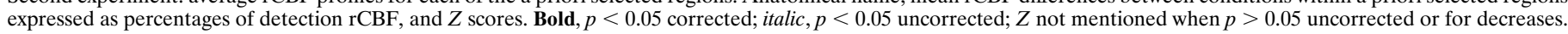

flow levels at extreme ends, whereas the levels during divided attention (ldro and lord) lay midway between these extremes. Obviously, if such an effect were obtained in regions defined by the contrast between leftward and rightward conditions in the second experiment, it might have been an artifact induced by selection bias. Critically, the regions studied were defined a priori in an independent experiment, and, therefore, the average $\mathrm{rCBF}$ level during divided attention most probably reflects a true physiological substrate for divided attention.

\section{Parietal cortex}

Dividing attention between two objects had no effect on superior parietal regions (Table 6).

\section{DISCUSSION}

\section{Single discriminations: direction-insensitive regions}

The activations during single peripheral discriminations $(p<0.2)$ that were not influenced by the direction of attention were the right superior parietal lobule, the intraparietal sulcus bilaterally, the right upper and lower premotor cortex, the anterior cingulate, and the left pulvinar (Fig. $2 A$ ).

Earlier neuropsychological (Posner et al., 1987; Posner and Petersen, 1990) and PET experiments concerning shifting (Corbetta et al., 1993) or maintaining of peripheral attention (Vandenberghe et al., 1996) convincingly demonstrated that the parietal cortex fulfills a function in peripheral orienting of attention. Given the conventional role of parietal cortex in controlling the attentional focus (Posner and Petersen, 1990), a clear expectation might have been that parietal activity, in as far as it reflects peripheral orienting, would be modulated by direction of attention. Despite the strong parietal activations observed in some of our other subtractions, no such modulation was observed.

It is worth noting that two earlier PET studies of visuospatial attention also failed to reveal any parietal direction sensitivity (Heinze et al., 1994; Woldorff et al., 1996). In both of these studies the relevant stimulus was presented at a fixed peripheral location with distractors present in the ignored hemifield, as in our experiment. In a third study there was some trend toward higher parietal activation on the side opposite to the attended hemifield
(Corbetta et al., 1993). Even in this study, however, parietal activation was clearly bilateral, and, in fact, differences between leftward and rightward attention conditions were not demonstrated directly in a statistical way. Furthermore, experimental differences could account for the different results. For instance, the study by Corbetta et al. (1993) required systematic attention shifts among many locations within the selected hemifield. One key feature of the hemineglect syndrome after parietal lesions is a disengagement deficit that is restricted mainly to the contralateral visual hemifield (Posner et al., 1987; Posner and Petersen, 1990). This deficit is manifest when attention has to be shifted but is less obvious when attention remains on the cued location. One interpretation of all these results is that superior parietal activity depends on the attended hemifield only under certain shifting conditions, possibly depending on whether distractors are presented in the ignored hemifield. Whether or not this hypothesis is confirmed, it seems clear that there is little lateralization during sustained leftward or rightward attention in the presence of bilateral visual stimulation.

It remains to be seen what cognitive processes are associated with the direction-insensitive parietal activity we observed. In a previous study parietal activation was associated with attention to peripheral targets rather than to central targets (Vandenberghe et al., 1996), arguing for a role in the attentive processing of peripheral events. On the other hand, activation of the same or a nearby region with PET during nonspatial tasks indicates that parietal cortex also fulfills a more general role in maintaining cognitive readiness or alertness (Pardo et al., 1991; Coull et al., 1996). More work will be needed to discriminate such alternatives.

Other direction-insensitive components of the general peripheral selective-attention network (see Table 3) were left pulvinar, right upper and lower premotor cortex, and right anterior cingulate. Structures near or identical to the premotor activations described have been reported by others studying covert (Corbetta et al., 1993) or overt orienting (Anderson et al., 1994; Sweeney et al., 1996). In the absence of any modulation by our current (Table 3) or previous (Vandenberghe et al., 1996) experimental manipulations, our data do not help us 
to specify further the precise contribution of these regions to covert peripheral attention.

\section{Single discriminations: direction-sensitive regions}

The left lateral frontal and right ventral occipital cortex were strongly influenced by the direction of attention: left frontal $\mathrm{rCBF}$ was higher when attention was directed ipsilaterally (Figs. $2 B, 3 B$, Tables 4, 6), and right ventral occipital $\mathrm{rCBF}$ was higher when attention was directed contralaterally (Fig. $2 B$, Tables 4, 6). A complementary (Table 4) but less consistent (Table 6) effect was found during rightward attention in the right inferior frontal sulcus (Fig. 3D). The right occipital modulation is in agreement with earlier ERP (Mangun et al., 1993; Heinze et al., 1994) and PET reports (Heinze et al., 1994), although these studies also found complementary effects in the left occipital lobe. Left-sided occipital effects of direction of attention were entirely absent in our experiments.

Our two experiments demonstrate unequivocally that left lateral frontal blood flow is sensitive to the direction of attention; it is higher during leftward than during rightward attention. The contrasts revealing this effect are narrowly matched for all processes except for direction of attention. These results were unexpected. The ipsilateral localization gives us a possible clue regarding the underlying spatial attentional process. Lateral frontal lesions can give rise to an imbalance in unilateral orienting, and lateral frontal patients may show increased orienting to the contralateral hemispace in covert attention (Mennemeier et al., 1994), oculomotor tasks (Guitton et al., 1985; Butter et al., 1988), and manual tasks (Kwong and Heilman, 1991), although contralateral neglect also has been described (Heilman and Valenstein, 1972). Lateral frontal lesions, including the frontal eye fields, also can lead to increased latencies of ipsilateral reflexive saccades (Henik et al., 1994). Several hypotheses have been put forward. Ipsilesional systems that orient attention to the contralesional side may be released from tonic inhibition exerted by the frontal cortex (Kwong and Heilman, 1991). Alternatively, contralesional systems that orient attention to the ipsilesional side may be deprived from the facilitation normally exerted by the frontal cortex on the lesioned side (Henik et al., 1994). These lesion data and the current findings require us to postulate a model in which covert orienting results from the balance among inhibitory, disinhibitory, and facilitatory influences within a distributed attentional system, including the lateral frontal cortex. The complex interplay among different components of such a system may be reminiscent of much better known oculomotor (Wallace et al., 1989, 1990) and motor systems (Alexander et al., 1986). Within such a model the absolute sign of the frontal blood flow difference is hard to interpret and may reflect inhibitory, disinhibitory, and facilitatory influences. At the current state of knowledge, several of these mechanisms could account equally well for changes in the balance between leftward and rightward attentional biases.

\section{Dual discrimination}

Behavioral data confirmed the efficiency with which we divide attention over several features of a single object (see Table 1) (Duncan, 1984, 1993). As a first underlying physiological mechanism, activations present during attention for single features of peripheral objects were enhanced during attention to two features. Second, activity in areas sensitive to the direction of spatial attention was enhanced when subjects attended to more features in the favored hemifield. The current experiments did not provide us with evidence in favor of our third a priori hypothesis, predicting parallel activation of featurespecific processing areas. This may be attributable mainly to the limited segregation of displacement and orientation processing, as revealed in the current $\mathrm{rCBF}$ data.

\section{Dual discrimination: enhancement in direction-insensitive regions}

The components of the peripheral attention circuit that were sensitive to the number of attended attributes, but not to the direction of attention, were the superior parietal lobules and the intraparietal sulci and the anterior cingulate.

When subjects attended to two attributes concurrently, parietal activity was enhanced, most prominently so in the left superior parietal lobule. This marked enhancement (Figs. 2, 3C) exceeded the summed responses obtained during the component single discriminations. It could reflect the higher demands that concurrent discriminations put on peripheral attention resources in comparison with single discriminations, enabling the subjects to maintain unchanged performance parameters. Although the right-hemispheric components of the peripheral selective-attention network are already involved even in less demanding peripheral selective-attention tasks (Fig. $2 A$ ), symmetrical left-hemispheric areas become more active when the attentional load increases (Fig. $2 C$ ).

Similarly, the anterior cingulate activation obtained during single discrimination was enhanced further during dual discrimination (see Table 5). The absence of any modulation by our other task variables in this region leaves open a number of possible interpretations. For instance, anterior cingulate activation could be attributed to divided attention (Corbetta et al., 1991), dual task performance (Fletcher et al., 1995) or differences in response selection requirements (Deiber et al., 1991).

\section{Dual discrimination: enhancement in direction-sensitive regions}

The left lateral frontal region we describe shows a combined effect of the number of attended attributes (Figs. 2C, 3C, Table 5) and direction of attention (Figs. $2 B, 3 B$, Table 6 ). This combined effect is observed in its prefrontal as well as its lower premotor component (Table 5).

If we considered the left lateral frontal activation during dual, as compared with single, discrimination in isolation from the strong effect of direction of spatial attention, we might attribute it to linguistic or general cognitive (D'Esposito et al., 1995) differences between the dual and single discrimination tasks used. We cannot exclude that the extensive activation observed includes subregions involved in these processes. These alternative hypotheses only fall short in explaining the effect of direction of attention observed in the overall region (Table 6) and in its most significant components (Table 5). The dual discrimination conditions with a left or with a right object were matched strictly in linguistic and working memory aspects. The combined effect of the number of attended attributes and direction of attention can be explained most parsimoniously as indicative for a spatial attentional process and may reflect the higher intensity with which spatial attention is directed toward the attended hemifield and away from the ignored hemifield during dual, as compared with single, discrimination.

\section{Dividing attention over objects in opposite hemifields}

When subjects perform two feature discriminations concurrently, accuracy declines when the two features belong to two different objects (Duncan, 1984, 1993). This was confirmed by the behavioral results obtained in the second experiment (see Table 2). The PET results suggest a physiological homolog of this behavioral cost. When subjects divided attention over the two hemifields, levels of blood flow in left lateral frontal and right ventral occipital cortex were intermediate between the extreme values obtained during exclusively leftward or rightward attention (see Table 6). The rCBF difference between divided and focused attention is in agreement with the above 
interpretation that the frontal effects could reflect the intensity with which attention is directed ipsilaterally and away from the contralateral hemifield. The right occipital effects could reflect enhanced processing of the contralateral stimulus during focused, as compared with divided, attention.

\section{Conclusion}

In conclusion, these experiments provide us with a physiological expression of how the brain copes efficiently with dividing attention over features of single objects. First, left superior parietal and anterior cingulate activation during single discriminations are strongly enhanced during dual discrimination, regardless of the direction of attention. Second, a left lateral frontal region that is activated when attention is toward the ipsilateral field is even more active when more features are attended within the ipsilateral visual field. We hypothesize that both activations reflect the increased intensity with which attention is directed toward a peripheral object.

On the other hand, the behavioral loss incurred when we divide our attention between objects in opposite hemifields is paralleled by a very specific occipitofrontal pattern (see Table 6): whereas activity in this set of regions reaches its extreme levels when subjects focus exclusively on the left or on the right object, their activity lies in between when the subjects divide their attention. Whereas the two extreme brain states during focused attention may relate to the behavioral gain of focusing attention on one single object, the "compromise" state possibly could relate to the behavioral loss observed when we divide attention over more objects.

\section{REFERENCES}

Alexander G, Long M, Strick P (1986) Parallel organization of functionally segregated circuits linking basal ganglia and cortex. Annu Rev Neurosci 9:357-381.

Anderson T, Jenkins I, Brooks D, Hawken M, Frackowiak R, Kennard C (1994) Cortical control of saccades and fixation in man: a PET study. Brain 117:1073-1084.

Butter C, Rapcsak S, Watson R, Heilman K (1988) Changes in sensory inattention, directional akinesia, and release of the fixation reflex following a unilateral frontal lesion: a case report. Neuropsychologia 26:533-545.

Corbetta M, Miezin F, Dobmeyer S, Shulman G, Petersen S (1991) Selective and divided attention during visual discriminations of shape and color and speed: functional anatomy by positron emission tomography. J Neurosci 11:2383-2402.

Corbetta M, Miezin F, Shulman G, Petersen S (1993) A PET study of visuospatial attention. J Neurosci 13:1202-1226.

Coull J, Frith C, Frackowiak RSJ, Grasby P (1996) A fronto-parietal network for rapid visual information processing: a PET study of sustained attention and working memory. Neuropsychologia 34:1085-1095.

Deiber MP, Passingham R, Colebatch K, Nixon P, Frackowiak RSJ (1991) Cortical areas and the selection of movement: a study with positron emission tomography. Exp Brain Res 84:393-402.

Desimone R, Duncan J (1995) Neural mechanisms of selective visual attention. Annu Rev Neurosci 18:193-222.

D'Esposito M, Detre J, Alsop D, Shin R, Atlas S, Grossman M (1995) The neural basis of the central executive system of working memory. Nature 378:279-281.

Duncan J (1984) Selective attention and the organization of visual information. J Exp Psychol Gen 113:501-517.

Duncan J (1993) Coordination of what and where in visual attention. Perception 22:1261-1270.

Egly R, Driver J, Rafal R (1994) Shifting visual attention between objects and locations: evidence from normal and parietal lesion subjects. J Exp Psychol Gen 123:161-177.

Fletcher P, Frith C, Grasby P, Shallice T, Frackowiak RSJ, Dolan R (1995) Brain systems for encoding and retrieval of auditory-verbal memory: an in vivo study in humans. Brain 118:401-416.
Friston KJ, Ashburner J, Poline J, Frith C, Heather J, Frackowiak RSJ (1995a) Spatial realignment and normalization of images. Hum Brain Mapp 2:165-189.

Friston KJ, Holmes A, Worsley K, Poline J, Frith C, Heather J, Frackowiak RSJ (1995b) Statistical parametric maps in functional imaging: a general approach. Hum Brain Mapp 2:189-210.

Guitton D, Buchtel H, Douglas R (1985) Frontal lobe lesions in man cause difficulties in suppressing reflexive glances and in generating goal-directed saccades. Exp Brain Res 58:455-472.

Haxby J, Horwitz B, Ungerleider L, Maisog J, Pietrini P, Grady C (1994) The functional organization of human extrastriate cortex: a PET-rCBF study of selective attention to faces and locations. J Neurosci 14:6336-6353.

Heilman K, Valenstein E (1972) Frontal lobe neglect in man. Neurology 22:660-664.

Heinze H, Mangun G, Burchert W, Hinrichs H, Scholzan M, Munte T, Gos A, Scherg M, Johannes S, Hundeshagen H, Gazzaniga MS, Hillyard SA (1994) Combined spatial and temporal imaging of brain activity during visual selective attention in humans. Nature 372:543-546.

Henik A, Rafal R, Rhodes D (1994) Endogenously generated and visually guided saccades after lesions of the human frontal eye fields. J Cognit Neurosci 6:400-411.

Kwong S, Heilman K (1991) Ipsilateral neglect in a patient following a unilateral frontal lesion. Neurology 41:2001-2004.

Mangun R, Hillyard SA, Luck S (1993) Electrocortical substrates of visual selective attention. In: Attention and performance, Vol XIV (Meyer D, Kornblum S, eds), pp 219-243. Cambridge, MA: MIT.

Mennemeier M, Chatterjee A, Watson R, Wertman E, Carter L, Heilman K (1994) Contributions of the parietal and frontal lobes to sustained attention and habituation. Neuropsychologia 32:703-716.

Pardo J, Fox P, Raichle M (1991) Localization of a human system for sustained attention by positron emission tomography. Nature 349:61-64.

Poline JB, Vandenberghe R, Holmes A, Friston KJ, Frackowiak RSJ (1996) Reproducibility of PET activation studies: lessons from a multicenter European experiment. NeuroImage 4:34-54.

Posner M, Petersen S (1990) The attention system of the human brain. Annu Rev Neurosci 13:25-42.

Posner M, Walker J, Friedrich F, Rafal R (1987) How do the parietal lobes direct covert attention? Neuropsychologia 25:135-145.

Shulman G, Buckner R, Corbetta M, Miezin F, Raichle M, Petersen S (1996) Consistent cortical blood flow decreases during active visual tasks relative to passive viewing. NeuroImage 3:197.

Sweeney J, Mintun M, Kwee S, Wiseman M, Brown D, Rosenberg D, Carl J (1996) Positron emission tomography study of voluntary saccadic eye movements and spatial working memory. J Neurophysiol 75:454-468.

Talairach J, Tournoux P (1988) Co-planar stereotaxic atlas of the human brain. New York: Thieme.

Vandenberghe R, Dupont P, De Bruyn B, Bormans G, Michiels J, Mortelmans L, Orban GA (1996) The influence of stimulus location on the brain activation pattern in detection and orientation discrimination. Brain 119:1263-1276.

Van Voorhis S, Hillyard SA (1977) Visual evoked potentials and selective attention to points in space. Percept Psychophys 22:54-62.

Vecera S, Farah M (1994) Does visual attention select objects or locations? J Exp Psychol Gen 123:146-160.

Wallace S, Rosenquist A, Sprague J (1989) Recovery from cortical blindness mediated by destruction of nontectotectal fibers in the commissure of the superior colliculus in the cat. J Comp Neurol 284:429-450.

Wallace S, Rosenquist A, Sprague J (1990) Ibotenic acid lesions of the lateral substantia nigra restore visual orientation behavior in the hemianopic cat. J Comp Neurol 296:222-252.

Woldorff M, Fox P, Matzke M, Veeraswamy S, Jerabek P, Martin C (1995) Combined PET and ERP study of sustained visual spatial attention and visual target detection. Hum Brain Mapp 2:49.

Woldorff M, Fox P, Matzke M, Lancaster J, Veeraswamy S, Zamarripa F, Seabolt M, Glass T, Gao J, Martin C, Jerabek P (1996) Visual spatial attention: integration of PET and ERP data. NeuroImage 3:242. 